Pacific Journal of Mathematics

ALMOST DIFFEOMORPHISMS OF MANIFOLDS 


\title{
ALMOST DIFFEOMORPHISMS OF MANIFOLDS
}

\author{
R. De SAPIO
}

Let $f: M^{n} \rightarrow X^{n}$ be a homotopy equivalence between two closed, differentiable (of class $C^{\infty}$ ) $n$-manifolds such that $f$ induces, from the stable normal bundle $V_{k}\left(X^{n}\right)$ of $X^{n}$ in the $(n+k)$-sphere $S^{n+k}$, a bundle over $M^{n}$ that is equivalent to the stable normal bundle $V_{k}\left(M^{n}\right)$ of $M^{n}$ in $S^{n+k}$. Then it is found that the disjoint union $X^{n} \cup M^{n}$ bounds a differentiable $(n+1)$ manifold $W^{n+1}$ with a retraction $r: W^{n+1} \rightarrow X^{n}$ such that the restriction $r \mid M^{n}$ is equal to the given homotopy equivalence $f$. Furthermore, let $n=2 q-1$ or $2 q$, where $q \geqq 3$, and suppose that $X^{n}$ is simply connected if $n=2 q-1$, and that $X^{n}$ is 2-connected if $n=2 q$. Then, if the restrictions of the bundles $r^{*} V_{k}\left(X^{n}\right)$ and $V_{k}\left(W^{n+1}\right)$ to the $(q-1)$-skeleton of $W^{n+1}$ are equivalent, where $r^{*} V_{k}\left(X^{n}\right)$ is the bundle induced by $r: W^{n+1} \rightarrow X^{n}$ and $V_{k}\left(W^{n+1}\right)$ is the stable normal bundle of $W^{n+1}$ in $S^{n+k}$, then $M^{n}$ and $X^{n}$ are diffeomorphic up to a point. In particular, $M^{n}$ and $X^{n}$ are homeomorphic.

It was S. P. Novikov who first gave sufficient conditions for homotopically equivalent, closed, simply connected $n$-manifolds $M^{n}$ and $X^{n}$ to be homeomorphic, provided that $n \geqq 5$. In this paper one of his sufficient conditions is replaced by a weaker condition. The other condition of Novikov is assumed here, namely that the given homotopy equivalence $f: M^{n} \rightarrow X^{n}$ has the property that the induced bundle $f^{*} V_{k}\left(X^{n}\right)$ is equivalent to $V_{k}\left(M^{n}\right)$.

The homeomorphism obtained here is actually an almost diffeomorphism (as was obtained by Novikov) in the following sense: an almost diffeomorphism of closed $n$-manifolds $M^{n}$ and $X^{n}$ is a homeomorphism $g: M^{n} \rightarrow X^{n}$ that is a diffeomorphism except possibly at a single point. For $n \neq 3,4$ this is equivalent to the statement that there is a homotopy $n$-sphere $V^{n}$ such that $M^{n}$ is diffeomorphic to the connected sum $X^{n} \# V^{n}$. Of course Novikov's conclusion is stronger than the one obtained here in that he concludes that the homotopy $n$-sphere $V^{n}$ bounds a parallelizable manifold, which may not be the case here. This is due to the fact that we have removed one of Novikov's conditions on the homotopy equivalence.

The reason for removing this condition is clear from the following example. Let $n=9$ or 13 and let $M^{n}$ be the tangent $(n-1) / 2$-sphere bundle to the $(n+1) / 2$-sphere. Let $X^{n}$ be the connected sum of $M^{n}$ with a homotopy $n$-sphere that does not bound a parallelizable manifold; such homotopy $n$-spheres exist at least for $n=9,13$, and 15 (see [5]). Then $M^{n}$ and $X^{n}$ have the same homotopy type (they are almost 
diffeomorphic) and have trivial stable normal bundles in a high dimensional $(n+k)$-sphere $S^{n+k}$. But for any homotopy $n$-sphere $V^{n}$ that bounds a parallelizable manifold, the manifolds $M^{n}$ and $X^{n} \# V^{n}$ are never diffeomorphic (see [2]). A more trivial example of this would be to take $M^{n}=S^{n}$ the standard $n$-sphere, and to take $X^{n}$ to be a homotopy $n$-sphere that does not bound a parallelizable manifold, say for $n=9,13$, or 15 .

In other words, what is desired is a necessary and sufficient condition for two closed $n$-manifolds $M^{n}$ and $X^{n}$ of the same homotopy type to be almost diffeomorphic. A necessary condition is that there exists a homotopy equivalence $f: M^{n} \rightarrow X^{n}$ such that $f$ induces, from the stable normal bundle of $X^{n}$ in $S^{n+k}$, a bundle over $M^{n}$ that is equivalent to the stable normal bundle of $M^{n}$ in $S^{n+k}$. This follows immediately from the fact that homotopy $n$-spheres have trivial stable normal bundles in $S^{n+k}$ (i.e. they are $\pi$-manifolds). However, in general this necessary condition does not appear to be sufficient, although it is in fact a sufficient condition for almost diffeomorphism in either one of the following two cases:

1. $n=2 q>4$ and $M^{n}$ is ( $\left.q-1\right)$-connected (see [6]).

2. $n=2 q+1$, where $q$ is even and $M^{n}$ is $(q-1)$-connected and stably parallelizable such that $H_{q}\left(M^{n}\right)$ is cyclic (see [2]).

In general the necessary condition above does imply the existence of a cobordism between $M^{n}$ and $X^{n}$ with a certain nice property. Then in order to conclude that $M^{n}$ and $X^{n}$ are almost diffeomorphic we make an assumption about this cobordism. In all honesty, it must be said that this assumption is really quite unsatisfactory at least because it is not a necessary condition in general.

Differentiable (or smooth) will mean of class $C^{\infty}$ and all manifolds are assumed to be differentiable, compact, and oriented (except as noted).

1. The cobordism. For a space $X$ and integer $n \geqq 0$ there is defined the $n$-dimensional unoriented bordism group $\mathfrak{R}_{n}(X)$ as developed by Conner and Floyed [1]. The abelian group $\mathfrak{N}_{n}(X)$ consists of bordism classes $\left[M^{n}, f\right]_{2}$ of singular $n$-manifolds $\left(M^{n}, f\right)$, where $M^{n}$ is a closed (possibly nonorientable) $n$-manifold and $f: M^{n} \rightarrow X$ is a map.

Suppose that $M^{n}$ and $X^{n}$ are closed $n$-manifolds and that $f: M^{n} \rightarrow$ $X^{n}$ is a map. Then the singular $n$-manifold $\left(M^{n}, f\right)$ determines an element $\left[M^{n}, f\right]_{2} \in \mathfrak{R}_{n}\left(X^{n}\right)$. Now the identity map $i d: X^{n} \rightarrow X^{n}$ also determines an element $\left[X^{n}, i d\right]_{2} \in \mathfrak{N}_{n}\left(X^{n}\right)$ and in general $\left[M^{n}, f\right]_{2} \neq$ $\left[X^{n}, i d\right]_{2}$. However, Conner and Floyd [1] give a necessary and sufficient condition for $\left[M^{n}, f\right]_{2}=\left[X^{n}, i d\right]_{2}$ in terms of the so called Whitney numbers of a map. If it is true that $\left[M^{n}, f\right]_{2}=\left[X^{n}, i d\right]_{2}$, then by definition there is a compact $(n+1)$-manifold $W^{n+1}$, possibly 
nonorientable, with boundary the disjoint union $X^{n} \cup M^{n}$ and a map $r: W^{n+1} \rightarrow X^{n}$ such that $r \mid M^{n}=f$ and $r \mid X^{n}$ is the identity on $X^{n}$. That is, $r$ is a retraction of $W^{n+1}$ onto $X^{n}$.

We have a map $f: M^{n} \rightarrow X^{n}$ of closed $n$-manifolds. Let $\mu_{n} \in H_{n}\left(M^{n} ; Z_{2}\right)$ be the fundamental class. Then for any $\gamma \in H^{n}\left(M^{n} ; Z_{2}\right)$ there is defined the Kronecker index $\left\langle\gamma, \mu_{n}\right\rangle \in Z_{2}$. Now let $w_{0}=1, w_{1}, \cdots, w_{n}$ denote the Stiefel-Whitney classes of $M^{n}$. Choose any cohomology class $c^{m} \in H^{m}\left(X^{n} ; Z_{2}\right)$; then for any partition $i_{1}+\cdots+i_{k}=n-m$ the product $w_{i_{1}} \cdots w_{i_{k}} f^{*}\left(c^{m}\right)$ lies in $H^{n}\left(M^{n} ; Z_{2}\right)$ and hence $\left\langle w_{i_{1}} \cdots w_{i_{k}} f^{*}\left(c^{m}\right)\right.$, $\left.\mu_{n}\right\rangle \in Z_{2}$ is defined. Following [1], this integer $\bmod 2$ is called a Whitney number of the map $f$ associated with $c^{m} \in H^{m}\left(X^{n}: Z_{2}\right)$. We remark that the Whitney numbers are also defined when $M^{n}$ is nonorientable in exactly the same way. There are also defined the Whitney numbers of the map $i d: X^{n} \rightarrow X^{n}$. Now a result of $[1, T h$. 17.2, p. 47] asserts that $\left[M^{n}, f\right]_{2}=\left[X^{n}, i d\right]_{2}$ if and only if $f: M^{n} \rightarrow X^{n}$ and $i d: X^{n} \rightarrow X^{n}$ have the same Whitney numbers.

Notation. Let $V_{k}(X)$ denote the stable normal bundle of a manifold $X$ in euclidean space.

Lemia 1. Let $f: M^{n} \rightarrow X^{n}$ be a homotopy equivalence such that the induced bundle $f^{*} V_{k}\left(X^{n}\right)$ is equivalent to $V_{k}\left(M^{n}\right)$. Then there is an $(n+1)$-manifold $W^{n+1}$ with boundary the disjoint union $X^{n} \cup M^{n}$ and a retraction $r: W^{n+1} \rightarrow X^{n}$ such that $r \mid M^{n}=f$.

Proof. Since $f^{*} V_{k}\left(X^{n}\right)$ is equivalent to $V_{k}\left(M^{n}\right), f$ induces the stable tangent bundle of $M^{n}$ from the stable tangent bundle of $X^{n}$. Hence by the naturality of the Stiefel-Whitney classes we have

$$
f^{*}\left(\widetilde{w}_{i}\right)=w_{i},
$$

where $\widetilde{w}_{i}$ is the $i$-th Stiefel-Whitney class of $X^{n}$. By previous remarks it is sufficient to show that $f: M^{n} \rightarrow X^{n}$ and $i d: X^{n} \rightarrow X^{n}$ have the same Whitney numbers, as follows. If $\mu_{n} \in H_{n}\left(M^{n} ; Z_{2}\right)$ and $\tilde{\mu}_{n} \in H_{n}\left(X^{n} ; Z_{2}\right)$ are the fundamental classes, then

$$
f_{*}\left(\mu_{n}\right)=\tilde{\mu}_{n}
$$

since $f$ is a homotopy equivalence and hence, given $c^{m} \in H^{m}\left(X^{n} ; Z_{2}\right)$ and $i_{1}+\cdots+i_{k}=n-m$, we have

$$
\begin{aligned}
\left\langle w_{i_{1}} \cdots w_{i_{k}} f^{*}\left(c^{m}\right), \mu_{n}\right\rangle & =\left\langle\widetilde{w}_{i_{1}} \cdots \widetilde{w}_{i_{k}} c^{m}, f_{*} \mu_{n}\right\rangle \\
& =\left\langle\widetilde{w}_{i_{1}} \cdots \widetilde{w}_{i_{k}} c^{m}, \widetilde{\mu}_{n}\right\rangle
\end{aligned}
$$

as desired. 


\section{The condition.}

THEOREM. Let $f: M^{n} \rightarrow X^{n}$ be a homotopy equivalence of simply connected, closed n-manifolds such that $f^{*} V_{k}\left(X^{n}\right)$ is equivalent to $V_{k}\left(M^{n}\right)$. Then:

(a) The disjoint union $M^{n} \cup X^{n}$ bounds an $(n+1)$-manifold $W^{n+1}$ with a retraction $r: W^{n+1} \rightarrow X^{n}$ such that $r \mid M^{n}=f$.

(b) Let $r: W^{n+1} \rightarrow X^{n}$ be as in (a) and suppose that the restrictions of the bundles $r^{*} V_{k}\left(X^{n}\right)$ and $V_{k}\left(W^{n+1}\right)$ to the $[(n-1) / 2]$-skeleton of $W^{n+1}$ are equivalent. Then $M^{n}$ and $X^{n}$ are almost diffeomorphic for $n \geqq 5$, provided that $X^{n}$ is 2-connected when $n$ is even.

REMARK. Here $[x]$, where $x$ is a real number, denotes the greatest integer less than or equal to $x$. Furthermore, if $H_{*}\left(X^{n}\right)$ has torsion consisting entirely of elements of order two, then, by considering the Pontrjagin numbers of the map $f: M^{n} \rightarrow X^{n}$ (cf. [1, p. 48]), we may apply [1, Th. 17.5, p. 49] to conclude that the manifold $W^{n+1}$ of Lemma 1 (and hence of (a) in the theorem) may be taken to be orientable; hence by application of the method of Browder and Novikov as employed in the following proof, the manifold $W^{n+1}$ of (a) in the theorem may be taken to be simply connected.

Proof. For convenience write $n=2 q-1$ or $2 q, q \geqq 3$. In the first place if $r^{*} V_{k}\left(X^{n}\right)$ is equivalent to $V_{k}\left(W^{n+1}\right)$, then it can be shown that this condition is equivalent to the condition of Novikov and hence it follows from the theorem of Novikov that $M^{n}$ and $X^{n}$ are almost diffeomorphic. However, the assumptions made here are sufficient to insure that we may apply the technique of Browder and Novikov (see [6] or [8]) to kill the kernel (which is finitely generated at each stage) of $r_{*}: \pi_{i}\left(W^{n+1}\right) \rightarrow \pi_{i}\left(X^{n}\right)$ in dimensions $i<q$ by spherical modifications of $W^{n+1}$. It is only necessary to check that each element in the kernel of $r_{*}$ for $i<q$ is represented by a smooth embedding $S^{i} \rightarrow W^{n+1}$ with trivial normal bundle. The existence of the embedding is due to Whitney-Haefliger (since $n+1>2 i+1$ for $i<q$ ) and the fact that it has trivial normal bundle follows from an argument similar to one due to Milnor [7, Th. 3] (the assumption concerning the $(q-1)$-skeleton of $W^{n+1}$ plays a role here to conclude that $r^{*} V_{k}\left(X^{n}\right)$ and $V_{k}\left(W^{n+1}\right)$ are equivalent when restricted to an open submanifold of $W^{n+1}$ that contains the $(q-1)$-skeleton of $\left.W^{n+1}\right)$. Hence we may assume that $r_{*}: \pi_{i}\left(W^{n+1}\right) \rightarrow \pi_{i}\left(X^{n}\right)$ is an isomorphism for $i<q$ and therefore, from considering the exact homotopy sequence of the pair $\left(W^{n+1}, X^{n}\right)$, it follows that $\pi_{i}\left(W^{n+1}, X^{n}\right)=0$ for $i<q$. Since $r \mid M^{n}$ is a homotopy equivalence it follows similarly that $\pi_{i}\left(W^{n+1}, M^{n}\right)=0$ for $i<q$.

It follows from handlebody theory that $W^{n+1}$ may be obtained 
from $X^{n}$ (or $M^{n}$ ) by successively attaching a finite number of "handles" to $X^{n} \times I$ (or $M^{n} \times I$, where $I=[0,1]$ is the unit interval) or, what is the same thing, by performing a finite sequence of spherical modifications on $X^{n}$ (or $M^{n}$ ). The argument now splits up into two cases, $n=2 q-1$ and $n=2 q$. Both arguments use the fact that

$$
\pi_{i}\left(W^{n+1}, X^{n}\right)=\pi_{i}\left(W^{n+1}, M^{n}\right)=0, \quad i<q .
$$

Case 1. $n=2 q-1$. It follows from (1) and [9, p. 539] that $W^{n+1}$ is diffeomorphic to

$$
\left(X^{n} \times I\right) \bigcup_{\varphi}\left(\bigcup_{j=1}^{s} D_{j}^{q} \times D^{q}\right),
$$

where $\varphi: \bigcup_{j=1}^{s}\left(\partial D_{j}^{q} \times D^{q}\right) \rightarrow X^{n} \times 1$ is an embedding of $s$ copies of $\partial D^{q} \times D^{q}=S^{q-1} \times D^{q}\left(D^{q}\right.$ the unit $q$-disc in real $q$-space) disjointly in $X^{n} \times 1$ and where the handles $D_{j}^{q} \times D^{q}$ are attached along $X^{n} \times 1$ by means of $\varphi$. Now in the portion of the exact homotopy sequence

$$
\pi_{q}\left(W^{n+1}, X^{n}\right) \stackrel{\partial}{\longrightarrow} \pi_{q-1}\left(X^{n}\right) \stackrel{i_{*}}{\longrightarrow} \pi_{q-1}\left(W^{n+1}\right)
$$

where $i: X^{n} \rightarrow W^{n+1}$ is the inclusion and $i_{*}$ is an isomorphism, we see that $\partial$ is trivial and hence $\varphi \mid \partial D^{q} \times 0$ is homotopically trivial. Thus $\varnothing$ is homotopically trivial and, since $X^{n}$ is simply connected, it follows from the engulfing theorem of [4] that $\varphi\left(\bigcup_{j} \partial D_{j}^{q} \times 0\right)$ is contained in the interior of a combinatorial $n$-disc embedded in $X^{n} \times 1$. The boundary of this disc may be smoothed in $X^{n} \times 1$ to conclude that $\varphi\left(\bigcup_{j} \partial D_{j}^{q} \times D^{q}\right)$ is contained in a smooth $n$-disc in $X^{n} \times 1$. Hence $W^{n+1}$ is diffeomorphic to the boundary connected sum $\left(X^{n} \times I\right.$, $\left.X^{n} \times 1\right) \#\left(W_{2}^{n+1}, \partial W_{2}^{n+1}\right)$, where $W_{2}^{n+1}=D^{n+1} \bigcup_{\varphi}\left(\bigcup_{j=1}^{s} D_{j}^{q} \times D^{q}\right)$. That is, $W_{2}^{n+1}$ is a handlebody such that $\partial W_{2}^{n+1} \# X^{n}$ is deffeomorphic to $M^{n}$. It is well known that $\partial W_{2}^{n+1}$ is a simply connected $n$-manifold and since $X^{n}$ and $M^{n}$ have the same homotopy type, $H_{i}\left(\partial W_{2}^{n+1}\right)=0$ for $0<i<n$. Thus $\partial W_{2}^{n+1}$ is a homotopy $n$-sphere, as desired.

REMARK. I should like to thank M. Hirsch for informing me that the engulfing theorem of [4] could be applied here. I later discovered that this engulfing theorem could also be applied to the more complicated situation found in Case 2 below.

Case 2. $n=2 q$. It follows from (1) and [9] that there is a smooth embedding

$$
\varphi: \bigcup_{j=1}^{s}\left(\partial D_{j}^{q} \times D^{q+1}\right) \longrightarrow X^{n} \times 1
$$

that (by the same argument as that of Case 1) embeds $\bigcup_{j=1}^{s} \partial D_{j}^{q} \times D^{q+1}$ 
into the interior of an $n$-disc $D^{n}$ that is embedded in $X^{n} \times 1$, and there is a smooth embedding

$$
\psi: \bigcup_{i=1}^{t}\left(\partial D_{i}^{q+1} \times D^{q}\right) \longrightarrow\left(X^{n} \times 1\right) \# \partial W_{2}^{n+1},
$$

where $W_{2}^{n+1}=D^{n+1} \bigcup_{\varphi}\left(\bigcup_{j=1}^{s} D_{j}^{q} \times D^{q+1}\right)$, such that $W^{n+1}$ is diffeomorphic to the boundary connected sum

$$
\left[\left(X^{n} \times I, X^{n} \times 1\right) \#\left(W_{2}^{n+1}, \partial W_{2}^{n+1}\right)\right] \bigcup_{\psi}\left[\bigcup_{i=1}^{t} D_{i}^{q+1} \times D^{q}\right],
$$

with the handles $D_{i}^{q+1} \times D^{q}$ attached by means of $\psi$. We shall show that we may assume that $\psi$ embeds $\bigcup_{i} \partial D_{i}^{q+1} \times D^{q}$ into $\partial W_{2}^{n+1}$-point, from which it will follow that $W^{n+1}$ is diffeomorphic to $\left(X^{n} \times I\right.$, $\left.X^{n} \times 1\right) \#\left(N^{n+1}, \partial N^{n+1}\right)$, where

$$
N^{n+1}=W_{2}^{n+1} \bigcup_{\psi}\left(\bigcup_{i} D_{i}^{q+1} \times D^{q}\right),
$$

and hence $M^{n}$ is diffeomorphic to $X^{n} \# \partial N^{n+1}$; but, as in Case 1, $\partial N^{n+1}$ is a homotopy $n$-sphere.

Lemma 2. If $A$ and $B$ are simplicial complexes such that $A$ is simply connected and $B$ is $(q-1)$-connected, then $\pi_{i}(A \vee B)=$ $\pi_{i}(A)+\pi_{i}(B)$ for $1 \leqq i \leqq q$.

This is a well known result in homotopy theory and follows from the fundamental fact that

$$
\pi_{i}(A \vee B)=\pi_{i}(A)+\pi_{i}(B)+\pi_{i+1}(A \times B, A \vee B) \quad(i>1)
$$

and the relative Hurewicz theorem, noting that $H_{i+1}(A \times B, A \vee B)=0$ for $i \leqq q$. We shall apply this lemma to the case where

$$
A=X^{n} \times 1 \text {-point }, \quad B=\partial W_{2}^{n+1} \text {-point . }
$$

Since $A \vee B$ has the homotopy type of $\left(X^{n} \times 1\right) \# \partial W_{2}^{n+1}$ with a point removed, we shall write for convenience

$$
A \vee B=\left[\left(X^{n} \times 1\right) \neq \partial W_{2}^{n+1}\right] \text {-point . }
$$

We begin by showing that we can assume that $r \mid A \vee B$ is the identity on $A=X^{n} \times 1$-point and is, after changing the $q$-handles in the decomposition of $W^{n+1}$ if necessary, homotopically trivial on $B=\partial W_{2}^{n+1}$-point. It will then follow that the homomorphism

$$
\pi_{q}(A)+\pi_{q}(B)=\pi_{q}(A \vee B) \longrightarrow \pi_{q}(A)
$$

that is induced by $r \mid A \vee B$ is the identity on $\pi_{q}(A)$ and is zero on 
$\pi_{q}(B)$. We shall divide this argument into two steps. For convenience introduce the notation

$$
V_{1}^{n+1}=\left(X^{n} \times I\right) \bigcup_{\varphi}\left(\bigcup_{j} D_{j}^{q} \times D^{q+1}\right) ;
$$

then $\partial V_{1}^{n+1}$ is the disjoint union of the "lower" boundary $X^{n}=X^{n} \times 0$ and the "upper" boundary $Y_{1}^{n}=\left(X^{n} \times 1\right) \# \partial W_{2}^{n+1}$.

Step 1. Consider the portion of the exact homotopy sequence of the pair $\left(V_{1}^{n+1}, X^{n}\right)$ :

$$
0 \longrightarrow \pi_{q}\left(X^{n}\right) \underset{r_{*}}{\stackrel{i_{*}}{\rightleftarrows}} \pi_{q}\left(V_{1}^{n+1}\right) \underset{\theta}{\stackrel{h_{*}}{\rightleftarrows}} \pi_{q}\left(V_{1}^{n+1}, X^{n}\right) \longrightarrow 0,
$$

where $h_{*}$ is induced by the inclusion. Since $r$ is a retraction, this short exact sequence splits and $\theta$ is the unique homomorphism such that $r_{*} \theta$ is zero and $h_{*} \theta$ is the identity. By the relative Hurewicz theorem the $q$-cells $D_{j}^{q} \times 0$ attached by means of $\varphi$ represent a set of free generators $x_{j}(j=1, \cdots, s)$ for $\pi_{q}\left(V_{1}^{n+1}, X^{n}\right)$.

Let

$$
z_{j}=\theta\left(x_{j}\right) \quad(j=1, \cdots, s)
$$

and note that, by the theorems of Haefliger, each $z_{j}$ may be represented by a $q$-sphere $S_{j}^{q}$ that is smoothly embedded in the interior of $V_{1}^{n+1}$. We may suppose that the $S_{j}^{q}$ are pairwise disjoint and disjoint from $X^{n} \times[0,1 / 2]$. Now choose an $n$-disc that is smoothly embedded in $X^{n} \times \frac{1}{2}$ and then, for each $j=1, \cdots, s$, choose a smooth $(q-1)$-sphere within this $n$-disc in $X^{n} \times \frac{1}{2}$ such that these $(q-1)$-spheres are pairwise disjoint. For each $j=1, \cdots, s$ run a tube of the form $S^{q-1} \times I$ in the complement of $X^{n} \times[0,1 / 2]$ in $V_{1}^{n+1}$ from the $j$-th $(q-1)$-sphere in $X^{n} \times \frac{1}{2}$ to $S_{j}^{q}$, these tubes being pairwise disjoint, such that the result is the manifold $X^{n} \times \frac{1}{2}$ with $q$-cells attached. We may then thicken these $q$-cells so that the result is a manifold of the form

$$
V_{2}^{n+1}=\left(X^{n} \times[0,1 / 2]\right) \bigcup_{\lambda}\left(\bigcup_{j=1}^{s} D_{j}^{q} \times D^{q+1}\right)
$$

that is smoothly embedded in $V_{1}^{n+1}$; here $\lambda$ is a smooth embedding of $\bigcup_{j=1}^{s} \partial D_{j}^{q} \times D^{q+1}$ into an $n$-disc in $X^{n} \times \frac{1}{2}$. Now let

$$
B_{\jmath}^{q} \subset D^{n+1} \subset X^{n} \times[0,1 / 2]
$$

be a $q$-disc with $\partial B_{j}^{q}=\lambda\left(\partial D_{j}^{q} \times 0\right)$ such that $B_{j}^{q} \bigcup_{\lambda}\left(D_{j}^{q} \times 0\right)$ is a $q$-sphere embedded in 


$$
W_{3}^{n+1}=D^{n+1} \bigcup_{i}\left(\bigcup_{j} D_{j}^{q} \times D^{q+1}\right) \text {. }
$$

Clearly, we may suppose that the $q$-sphere $B_{j}^{q} \bigcup_{\lambda}\left(D_{j}^{q} \times 0\right)$ represents the element $z_{j}$ in $\pi_{q}\left(V_{1}^{n+1}\right)$. But then

$$
r_{*}\left(z_{j}\right)=r_{*} \theta\left(x_{j}\right)=0 \quad(j=1, \cdots, s)
$$

and so $r \mid B_{j}^{q} \bigcup_{\lambda}\left(D_{j}^{q} \times 0\right)$ is homotopically trivial for each $j$ and hence $r \mid W_{3}^{n+1}$-point is homotopically trivial. In particular, $r \mid \partial W_{3}^{n+1}$-point is homotopically trivial.

Step 2. We shall now show that we can isotopically deform the manifold $V_{2}^{n+1}$ onto $V_{1}^{n+1}$, the isotopy lying within $V_{1}^{n+1}$ and leaving fixed a neighborhood of $X^{n} \times 0$. It will then follow from Step 1 that, by replacing $W_{2}^{n+1}$ by $W_{3}^{n+1}$ if necessary, we can assume that $r \mid A \vee B$ is homotopically trivial on $B=\partial W_{2}^{n+1}$-point. The closure $C^{l}\left(V_{1}^{n+1}-V_{2}^{n+1}\right)$ of the complement of $V_{2}^{n+1}$ in $V_{1}^{n+1}$ is an $(n+1)$-manifold with simply connected "lower" boundary $Y_{2}^{n}=\left(X^{n} \times 1 / 2\right) \# \partial W_{3}^{n+1}$ and simply connected "upper" boundary $Y_{1}^{n}=\left(X^{n} \times 1\right) \# \partial W_{2}^{n+1}$. It is enough to show that $\mathrm{Cl}\left(V_{1}^{n+1}-V_{2}^{n+1}\right)$ is diffeomorphic to the product $Y_{2}^{n} \times I$; by the $h$-cobordism theorem of Smale this is equivalent to showing that $\mathrm{Cl}\left(V_{1}^{n+1}-V_{2}^{n+1}\right)$ is an $h$-cobordism between $Y_{2}^{n}$ and $Y_{1}^{n}$. Hence we must show that the groups $H_{i}\left(\mathrm{Cl}\left(V_{1}^{n+1}-V_{2}^{n+1}\right), Y_{k}\right)$ are zero for all $i$ and $k=1,2$. Now by excision, $H_{i}\left(\mathrm{Cl}\left(V_{1}^{n+1}-V_{2}^{n+1}\right), Y_{2}\right)$ is isomorphic to $H_{i}\left(V_{1}^{n+1}, V_{2}^{n+1}\right)$ and thus is zero if we show that the inclusion $\alpha: V_{2}^{n+1} \subset V_{1}^{n+1}$ induces isomorphisms of homology in all dimensions. The inclusion $\alpha$ induces a map of split short exact sequences of homology groups:

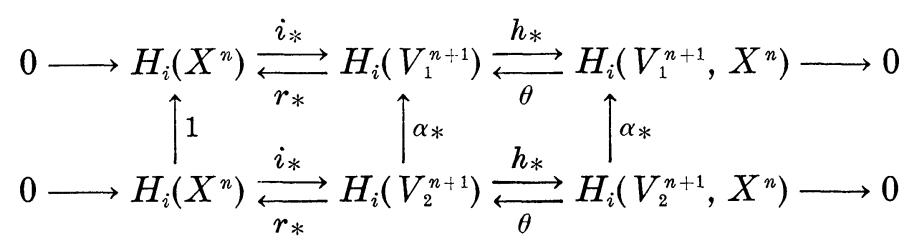

The maps $i_{*}, r_{*}, h_{*}, \theta$ of this commutative diagram are denoted by the same symbols as those used in the homotopy sequence of $\left(V_{1}^{n+1}, X^{n}\right)$ appearing above, and no confusion should arise because of this. Now $V_{2}^{n+1}$ and $V_{1}^{n+1}$ each have the homotopy type of $X^{n} \vee\left(\mathrm{V}_{j} S_{j}^{q}\right)$ and $\alpha: V_{2}^{n+1} \subset V_{1}^{n+1}$ is the identity on $X^{n}=X^{n} \times 0$. Hence $\alpha_{*}: H_{i}\left(X^{n}\right) \rightarrow$ $H_{i}\left(X^{n}\right)$ is the identity for all $i$ and $H_{i}\left(V_{2}^{n+1}, X^{n}\right)=H_{i}\left(V_{1}^{n+1}, X^{n}\right)=0$ for all $i \neq q$. In dimension $i=q$ we recall the set of free generators $\left\{x_{j} \mid j \neq 1, \cdots, s\right\}$ for $H_{q}\left(V_{1}^{n+1}, X^{n}\right)$ and that the element

$$
z_{j}=\theta\left(x_{j}\right) \in H_{q}\left(V_{1}^{n+1}\right)
$$


was represented by the smoothly embedded $q$-sphere $B_{j}^{q} \bigcup_{\lambda}\left(D_{j}^{q} \times 0\right)$ in $V_{1}^{n+1}$. But $B_{j}^{q} \bigcup_{2}\left(D_{j}^{q} \times 0\right)$ also represents an element $z_{j}^{\prime} \in H_{q}\left(V_{2}^{n+1}\right)$ such that $\alpha_{*}\left(z_{j}^{\prime}\right)=z_{j}$. Furthermore, the elements $h_{*}\left(z_{j}^{\prime}\right)(j=1, \cdots, s)$ form a set of free generators for $H_{q}\left(V_{2}^{n+1}, X^{n}\right)$ such that $\alpha_{*} h_{*}\left(z_{j}^{\prime}\right)=$ $h_{*} \alpha_{*}\left(z_{j}^{\prime}\right)=x_{j}$ for each $j=1, \cdots, s$ and hence $\alpha_{*}: H_{q}\left(V_{2}^{n+1}, X^{n}\right) \rightarrow$ $H_{q}\left(V_{1}^{n+1}, X^{n}\right)$ is an isomorphism. It follows that $\alpha_{*}: H_{i}\left(V_{2}^{n+1}\right) \rightarrow$ $H_{i}\left(V_{1}^{n+1}\right)$ is an isomorphism for all $i$ and hence, as noted above, $H_{i}\left(\mathrm{Cl}\left(V_{1}^{n+1}-V_{2}^{n+1}\right), Y_{2}\right)=0$ for all $i$. Now by successive applications of the Poincaré duality theorem and the universal coefficient theorem we see that $H_{i}\left(\mathrm{Cl}\left(V_{1}^{n+1}-V_{2}^{n+1}\right), Y_{1}\right) \approx H^{n+1-i}\left(\mathrm{Cl}\left(V_{1}^{n+1}-V_{2}^{n+1}\right), Y_{2}\right)$ is zero for all $i$, and thus it follows that $\mathrm{Cl}\left(V_{1}^{n+1}-V_{2}^{n+1}\right)$ is an $h$-cobordism between $Y_{2}^{n}$ and $Y_{1}^{n}$, as claimed.

We now turn to the study of the embedding $\psi$. Note that $\psi \mid \partial D_{i}^{q+1} \times 0$ represents an element in $\pi_{q}(A \vee B)$ and clearly $r \psi \mid \partial D_{i}^{q+1} \times 0$ is homotopically trivial. Therefore, since the homomorphism of (2) is the identity on $\pi_{q}(A)$ and zero on $\pi_{q}(B), \psi \mid \partial D_{i}^{q+1} \times 0$ is homotopic to a map $\partial D_{i}^{q+1} \times 0 \rightarrow B$ and thus $\psi$ is homotopic to a map

$$
\bigcup_{i=1}^{t}\left(\partial D_{i}^{q+1} \times 0\right) \longrightarrow B=\partial W_{2}^{n+1} \text {-point } .
$$

We should again like to apply the engulfing theorem of Hirsch and Zeeman [4] and conclude that $\psi$ is isotopic to an embedding with image in $B$. To this end note that $B$ is a $(q-1)$-connected $2 q$-manifold and hence has the homotopy type of a wedge of $q$-spheres $\mathrm{V}_{k} S_{k}^{q}$ embedded in $B$, each $q$-sphere $S_{k}^{q}$ smoothly embedded in $B$. Hence $\psi$ is homotopic to a map with image in $V_{k} S_{k}^{q}$ and it follows that in the language of [4], $\psi\left(\bigcup_{i} \partial D_{i}^{q+1} \times 0\right)$ is $\left(\mathbf{V}_{k} S_{k}^{q}\right)$-inessential. Since $A \vee B$ is 2-connected and $\pi_{i}\left(A \vee B, \bigvee_{k} S_{k}^{q}\right)=0$ for $i \leqq 2$ (recall that $A=X^{n} \times 1$-point is 2-connected by hypothesis) all of the conditions of the engulfing theorem [4] are satisfied and hence $\psi\left(\bigcup_{i} \partial D_{i}^{q+1} \times 0\right)$ is contained in a regular neighborhood of $\mathbf{V}_{k} S_{k}^{q}$ in

$$
A \vee B=\left[\left(X^{n} \times 1\right) \# \partial W_{2}^{n+1}\right] \text {-point . }
$$

It is well known that the boundary of this regular neighborhood may be smoothed in $A \vee B$ and hence we can assume that $\psi\left(\bigcup_{i} \partial D_{i}^{q+1} \times 0\right)$ is contained in a smooth regular neighborhood of $\bigvee_{k} S_{k}^{q}$ in $A \vee B$. Now

$$
\partial W_{2}^{n+1} \text {-interior of smooth } n \text {-disc } \subset \partial W_{2}^{n+1} \text {-point }=B
$$

is a smooth regular neighborhood of $\mathrm{V}_{k} S_{k}^{q}$ in $B$ and hence in $A \vee B$. But a result of [3, Th. 1] asserts that any smooth regular neighborhood may be isotopically deformed onto any other, and hence $\psi\left(\bigcup_{i} \partial D_{\imath}^{q+1} \times 0\right)$ may be isotopically deformed into $B$, completing the 
proof of the theorem.

COROLlary. Let $f: M^{n} \rightarrow X^{n}$ be a homotopy equivalence of simply connected n-manifolds such that $f^{*} V_{k}\left(X^{n}\right)$ is equivalent to $V_{k}\left(M^{n}\right)$. Then:

(a) The disjoint union $X^{n} \cup M^{n}$ bounds a $(n+1)$-manifold $W^{n+1}$ such that there is a retraction $r: W^{n+1} \rightarrow X^{n}$ with $r \mid M^{n}=f$.

(b) Let $W^{n+1}$ be as in (a) and suppose that $\pi_{i}\left(W^{n+1}, X^{n}\right)=0$ for $i \leqq[(n-1) / 2]$. Let $n \geqq 5$ and if $n$ is even, then assume that $X^{n}$ is 2-connected. Then $M^{n}$ and $X^{n}$ are almost diffeomorphic.

The corollary follows from the proof of the theorem.

REMARK. It can be shown that any homotopy $n$-sphere bounds a simply connected $(n+1)$-manifold $N^{n+1}$. In view of the above theorem the following question arises naturally: Can $N^{n+1}$ be taken to be $[(n-1) / 2]$-connected? If the answer is yes, then the condition on $W^{n+1}$ given in (b) of the theorem is necessary and sufficient for $M^{n}$ and $X^{n}$ to be almost diffeomorphic ( $n \geqq 5$ here).

However, the answer is negative for $n=2 q-1$ and $q \equiv$ $3,5,6,7(\bmod 8)$ since in this case $N^{n+1}$ would necessarily be parallelizable if it were $(n-1) / 2$-connected. This is so because $\pi_{q-1}(S O)=0$ for $q \equiv 3,5,6,7(\bmod 8)$. Hence the homotopy sphere obtained in (b) of the theorem bounds a parallelizable manifold for $n=2 q-1$ and $q \equiv 3,5,6,7(\bmod 8)$, and thus the condition given there is not in general necessary for $M^{n}$ and $X^{n}$ to be almost diffeomorphic.

\section{REFERENCES}

1. P. E. Conner and E. E. Floyd, Differentiable Periodic Maps, Ergebnisse der Mathematik und ihrer Grenzgebiete 33, Springer, Berlin-Göttingen-Heidelberg, 1964.

2. R. De Sapio, Actions of $\theta_{2 k+1}$, Michigan Math. J. 14 (1967), 97-100.

3. M. W. Hirsch, Smooth regular neighborhoods, Ann. of Math. 76 (1962), 524-530.

4. M. W. Hirsch and E. C. Zeeman, Engulfing, Bull. Amer. Math. Soc. 72 (1966), 113-115.

5. M. Kervaire and J. Milnor, Groups of homotopy spheres: I, Ann. of Math. 77 (1963), 503-537.

6. R. K. Lashof, Some theorems of Browder and Novikov on homotopy equivalent manifolds (mimeographed notes), The University of Chicago.

7. J. Milnor, A procedure for killing homotopy groups of differentiable manifolds, Proceedings of Symposia in Pure Math., A.M.S., Vol. III, 1961, 39-55.

8. C. T. C. Wall, An extension of results of Novikov and Browder, Amer. J. Math. 88 (1966), 20-32.

9. A. H. Wallace, A geometric method in differential topology, Bull. Amer. Math. Soc. 68 (1962), 533-542.

Received March 7, 1967. The preparation of this paper was sponsored in part by NSF Grant GP-5860. 


\section{PACIFIC JOURNAL OF MATHEMATICS}

\section{EDITORS}

\author{
H. ROYDEN \\ Stanford University \\ Stanford, California \\ R. R. Phelps \\ University of Washington \\ Seattle, Washington 98105
}

J. DUGUNDJI

Department of Mathematics

University of Southern California

Los Angeles, California 90007

RICHARD ARENS

University of California

Los Angeles, California 90024

\section{ASSOCIATE EDITORS}
E. F. BECKENBACH
B. H. NeumanN
F. WOLF
K. YosidA

\section{SUPPORTING INSTITUTIONS}

UNIVERSITY OF BRITISH COLUMBIA

CALIFORNIA INSTITUTE OF TECHNOLOGY

UNIVERSITY OF CALIFORNIA

MONTANA STATE UNIVERSITY

UNIVERSITY OF NEVADA

NEW MEXICO STATE UNIVERSITY

OREGON STATE UNIVERSITY

UNIVERSITY OF OREGON

OSAKA UNIVERSITY

UNIVERSITY OF SOUTHERN CALIFORNIA
STANFORD UNIVERSITY

UNIVERSITY OF TOKYO

UNIVERSITY OF UTAH

WASHINGTON STATE UNIVERSITY

UNIVERSITY OF WASHINGTON

AMERICAN MATHEMATICAL SOCIETY CHEVRON RESEARCH CORPORATION TRW SYSTEMS

NAVAL WEAPONS CENTER

Printed in Japan by International Academic Printing Co., Ltd., Tokyo, Japan 


\section{Pacific Journal of Mathematics}

\section{Vol. 26, No. $1 \quad$ November, 1968}

Efraim Pacillas Armendariz, Closure properties in radical theory......... 1

Friedrich-Wilhelm Bauer, Postnikov-decompositions of functors .......... 9

Thomas $\mathrm{Ru}-$ Wen Chow, The equivalence of group invariant positive definite

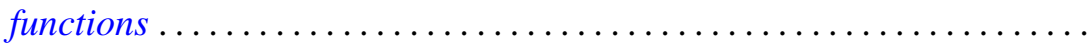

Thomas Allan Cootz, A maximum principle and geometric properties of

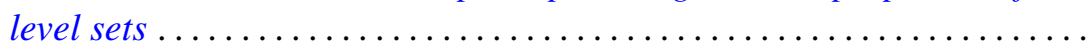

Rodolfo DeSapio, Almost diffeomorphisms of manifolds ............ 47

R. L. Duncan, Some continuity properties of the Schnirelmann density......

Ralph Jasper Faudree, Jr., Automorphism groups of finite subgroups of

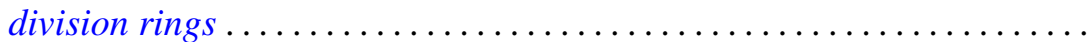

Thomas Alastair Gillespie, An invariant subspace theorem of $J$.

Feldman.........................................

George Isaac Glauberman and John Griggs Thompson, Weakly closed direct factors of Sylow subgroups .............................

Hiroshi Haruki, On inequalities generalizing a Pythagorean functional equation and Jensen's functional equation .....................

David Wilson Henderson, D-dimension. I. A new transfinite dimension.....

David Wilson Henderson, D-dimension. II. Separable spaces and

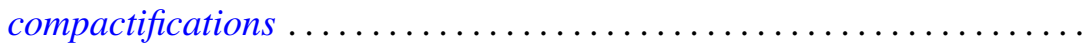

Julien O. Hennefeld, A note on the Arens products ............... 115

Richard Vincent Kadison, Strong continuity of operator functions ...

J. G. Kalbfleisch and Ralph Gordon Stanton, Maximal and minimal coverings of $(k-1)$-tuples by $k$-tuples.

Franklin Lowenthal, On generating subgroups of the Moebius group by pairs of infinitesimal transformations...

Michael Barry Marcus, Gaussian processes with stationary increments possessing discontinuous sample paths . .

Zalman Rubinstein, On a problem of Ilyeff ...

Bernard Russo, Unimodular contractions in Hilbert space. ...

David Lee Skoug, Generalized Ilstow and Feynman integrals...

William Charles Waterhouse, Dual groups of vector spaces . 